\title{
Medium-Term Outcome and CT Assessment of Lateral Foraminotomy at the Lumbosacral Junction in Dogs with Degenerative Lumbosacral Stenosis
}

\author{
Andrew John Worth ${ }^{1,2}$ Angela Hartman ${ }^{1} \quad$ Janis P. Bridges ${ }^{1} \quad$ Boyd R. Jones ${ }^{1} \quad$ Joe I. G. Mayhew ${ }^{1}$ \\ ${ }^{1}$ Institute of Veterinary, Animal and Biomedical Sciences, Massey \\ Address for correspondence Andrew John Worth, Centre for \\ University, Palmerston North, Manawatu, New Zealand \\ 2 Working Dog Centre, Massey University, Palmerston North, \\ Companion Animal Health, Massey University, Private Bag 11-222, \\ Palmerston North, Manawatu 4472, New Zealand \\ Manawatu, New Zealand \\ (e-mail: A.J.Worth@massey.ac.nz).
}

Vet Comp Orthop Traumatol 2018;31:37-43.

\begin{abstract}
Keywords

- lumbosacral

- cauda equine

- foraminotomy

- foraminal volume

- bone modelling

Objective This article aims to report the medium-term clinical outcome and assess persistence of enlargement of the lumbosacral lateral intervertebral neurovascular foramen using computed tomography (CT) volumetric analysis in dogs following lateral foraminotomy.

Materials Six dogs that underwent lumbosacral lateral foraminotomy on one or both sides were evaluated with CT prior to, immediately postoperatively $(n=2)$ and at 12 to 44 months of follow-up. Five out of six dogs had successful clinical outcomes with alleviation of pain and increased levels of activity, according to subjective assessment. Immediate postoperative CT volumetric analysis of the lateral intervertebral neurovascular foramina in two dogs indicated a 650 to $800 \%$ increase in volume in extension achieved by foraminotomy (four foramens). At subsequent follow-up, bone regrowth had occurred with reduction in foraminal volume, though in both dogs foraminal volume remained higher than preoperative values. Follow-up CT at a median of 24 months postoperatively indicated a mean $335 \%$ increase in volume of the lumbosacral lateral intervertebral neurovascular foramina in extension compared with the preoperative foraminal volume. The follow-up volume was substantially greater than the presurgical volume in four out of six dogs.

Clinical Significance In this limited case series, lateral foraminotomy achieved persistent enlargement of the lumbosacral lateral intervertebral neurovascular foramen in the medium term, but osseous regrowth at the site was demonstrated which may limit the effectiveness of lateral foraminotomy in the longer term. One of two working dogs had recurrent clinical signs that necessitated further surgery.
\end{abstract}

\section{Introduction}

Degenerative lumbosacral stenosis is characterized by an acquired narrowing of the vertebral canal, lateral intervertebral neurovascular foramina, or both at the lumbosacral junction, which results in compressive radiculopathy of one or more nerve roots of the cauda equina. ${ }^{1-4}$ Surgical management of degenerative lumbosacral stenosis is directed at decompression of the cauda equina, affected nerve roots, or both, or else stabilization of the lumbosacral junction. ${ }^{5-9}$ Dorsal compression of the cauda equina due to protrusion of the sacral lamina and hypertrophied interarcuate ligament received

May 2, 2017

accepted after revision

August 24, 2017
Copyright $\odot 2018$ Schattauer

DOI https://doi.org/ 10.3415/vcot-17-05-0061. ISSN 0932-0814. 
when the joint is in extension may resolve after decompression by dorsal laminectomy. ${ }^{1,10-13}$ Ventral compression of the cauda equina by protrusion of the dorsal annulus of the lumbosacral disc has traditionally been an indication for dorsal disc annulectomy and discectomy. ${ }^{5}$ The presence of ventral displacement of the sacrum (S1) relative to the seventh lumbar vertebrae (L7) and dynamic narrowing of the lumbosacral lateral intervertebral neurovascular foramina on extension are generally considered indications for distraction and stabilization. ${ }^{7,9,14,15}$ The presence of clinical signs indicative of $\mathrm{L} 7$ nerve root compression and narrowing of the lumbosacral lateral intervertebral neurovascular foramina with loss of fat signal around the L7 nerve roots is reported as an indication for nerve root decompression with a lateral foraminotomy. ${ }^{16}$ However, prospective comparative studies on which to make decisions for successful treatment of dogs with degenerative lumbosacral stenosis have not been undertaken. ${ }^{17}$

The lateral intervertebral neurovascular foramen is not a simple aperture but is a complex three-dimensional canal or tunnel. In humans, the L5-S1 neurovascular foramen is composed of an entrance, a middle and an exit zone which have been defined according to their location in regards to the pedicle and articular processes of $\mathrm{L} 5$ and $\mathrm{S} 1 .{ }^{18}$ While this terminology (entrance, middle and exit) has been applied to dogs, the L7 pedicle in dogs differs anatomically from that of humans, being thinner and orientated at a more oblique angle with a longer and narrower lateral recess. ${ }^{16,19}$

The lateral foraminotomy procedure reported by Gödde and Steffen ${ }^{16}$ may represent a significant improvement in the management of degenerative lumbosacral stenosis as it potentially allows greater decompression of the lumbosacral lateral intervertebral neurovascular foramen than can be achieved via a dorsal approach in the dog. To date, there has been only a single publication relating to the outcome of lateral lumbosacral foraminotomy in dogs. ${ }^{16}$ The developers of the technique undertook a retrospective evaluation of 20 dogs using a combination of veterinary re-examination 6 months postoperatively and a telephone interview with the owners of the dogs after the final follow-up. Unilateral or bilateral lateral lumbosacral foraminotomy was performed on each dog based on magnetic resonance imaging (MRI) findings of L7 nerve root compression. A limited dorsal laminectomy was performed concurrently in 11 of the 20 dogs. ${ }^{20}$ The decision to undertake concurrent dorsal laminectomy was based on MRI evidence of stenosis of the lumbosacral vertebral canal. The outcome was good to excellent in 19 of the 20 dogs at a mean follow-up of 15.2 months (range: 6-42 months). ${ }^{16}$ Recurrence was not noted in any of the 19 dogs that responded to surgery.

The study by Gödde and Steffen ${ }^{16}$ could not evaluate whether regrowth of bone occurred at the foraminotomy site and the mean follow-up was relatively short. Bone regrowth at the site of a dorsal laminectomy occurs in humans, ${ }^{21}$ and in an experimental study in healthy dogs, bone regrowth occurred at the site of an endoscopically assisted foraminotomy performed at the entrance and middle zones. ${ }^{19}$ By 12 weeks, there was no significant difference between foraminal area measurements of the control and operated sides despite significant enlargement noted immediately postoperatively. ${ }^{19}$ This raises the concern that bone regrowth at the site of a lumbosacral foraminotomy may reduce its long-term decompressive effect.

Assessment of the effect of foraminotomy requires an accurate method of measuring the size of the lumbosacral lateral intervertebral neurovascular foramen in vivo. The authors have recently published a method of volumetric assessment of the lumbosacral lateral intervertebral neurovascular foramen in dogs using $\mathrm{CT} .^{22}$

The aim of this observational study was to assess the outcome of lateral foraminotomy in a cohort of dogs with degenerative lumbosacral stenosis and confirmed narrowing of one or both lumbosacral lateral intervertebral neurovascular foramina. Volumetric analysis of CT images was used to measure the foraminal volume both immediately postoperatively in selected dogs and at least 12 months after foraminotomy. The authors sought to determine if bone regrowth occurs at the site of a lateral foraminotomy, as regrowth could be a limiting factor for the technique's long-term success in dogs with degenerative lumbosacral stenosis.

\section{Materials and Methods}

The study population consisted of six dogs diagnosed with degenerative lumbosacral stenosis that underwent lateral lumbosacral foraminotomy by a single specialist surgeon (A.J.W.) at the Massey University Veterinary Teaching Hospital between May 2009 and December 2015. During the same period, a total of nine dogs underwent lumbosacral foraminotomy for degenerative lumbosacral stenosis. Two of these developed progressive neuropathies inconsistent with degenerative lumbosacral stenosis and were euthanatized and one dog was lost to follow-up. All six dogs had clinical signs localized to the lumbosacral joint on physical and neurological examinations and had imaging findings consistent with L7 nerve root compression. Plain screening radiographs had been performed to rule out vertebral neoplasia and discospondylitis. A CT scan had been performed while dogs were sedated with medetomidine $(0.005 \mathrm{mg} / \mathrm{kg}$ IV) and butorphanol $(0.2 \mathrm{mg} / \mathrm{kg} \mathrm{IV})$ using a standard protocol (Phillips Brilliance helical 16-slice, Philips Healthcare, the Netherlands). The dogs were positioned in dorsal recumbency with the lumbosacral junction in maximal extension for the $\mathrm{CT}$ imaging. Soft-tissue algorithms were converted to three-dimensional renderings and assessed with tissue management filters. Volume measurements of the lumbosacral lateral intervertebral neurovascular foramina were performed according to a previously described protocol. ${ }^{22}$ All images had loss of fat signal in the lumbosacral lateral intervertebral neurovascular foramen consistent with compression of the $\mathrm{L} 7$ nerve on one or both sides and indications of lumbosacral disc degeneration and annular protrusion. The surgery was performed using a previously described procedure ${ }^{16}$ after attending a training course (Dorsolateral foraminotomy for DLS, Tomas Gödde, Tuttlingen, Germany, March 20-21, 2009). In dogs with central canal compression, either a traditional dorsal laminectomy 
including the caudal L7 lamina or a limited S1 dorsal laminectomy was performed. ${ }^{5,20}$

The initial two dogs were CT scanned again immediately postoperatively to assess the effect of surgery and rescanned at greater than 12-month follow-up using the same protocol as previously described. To minimize gas artefacts on $\mathrm{CT}$, the surgical site was flushed with saline prior to closure. Due to lack of available funding, the other four dogs were not scanned immediately postoperatively but were rescanned at a follow-up interval greater than 12-months postoperatively when additional research funding became available.

Postoperative instructions were standardized and included a requirement to restrict the dogs to a small run or a large cage for the first 6 weeks. In weeks 1 to 2, the dogs were allowed 5-minute leash walks for urination and defecation. This limited exercise increased to 10 minutes leash walking in weeks 3 and 4 and 15 minutes leash walking in weeks 5 and 6. Carprofen (Rimadyl, Zoetis NZ, Auckland, $2 \mathrm{mg} / \mathrm{kg}$ twice daily for 7 days) was administered to decrease inflammation and provide analgesia.

All dogs were reexamined 6 weeks after surgery to assess short-term complications. Dog 1 was CT scanned on two further occasions following the development of incidental discospondylitis at T13-L1. One of the two Police dogs (dog 6) was reexamined and rescanned by CT at 12 months postoperatively due to ongoing clinical signs preventing full return to work. The remaining three dogs were clinically examined, and a repeat CT scan was performed with informed owner consent using the same sedation/CT protocol as previously outlined. All owners were interviewed by telephone at least 12 months following surgery and coinciding with CT scanning. Using standardized questions, owners were asked to describe their dog's current mobility and relate that to the dog's clinical signs prior to surgery.

\section{Results}

Demographic and historical data of the six dogs are listed in - Table 1. Two dogs were Police German Shepherd dogs in active duty, and the remaining four were pet dogs. The median age of the dogs was 5 years (range: $3-8$ years). The median weight of the dogs was $32.25 \mathrm{~kg}$ (range: $14.5-39 \mathrm{~kg}$ ). Clinical signs had been present for a median of two and a half months prior to presentation, but there was a considerable range ( 2 months to 3 years). Clinical signs included pain on pressure over the lumbosacral junction, pelvic limb lameness, difficulty jumping, stiffness on rising and reluctance to exercise (see -Table 1). The imaging findings, surgical treatment and outcomes are listed in -Appendix Table 1. Three dogs (dogs 1, 2 and 6) had bilateral lateral foraminotomy combined with dorsal decompression. Two dogs (dogs 3 and 5) had unilateral lateral foraminotomy combined with dorsal laminectomy and one $\operatorname{dog}(\operatorname{dog} 4)$ had bilateral foraminotomy without dorsal laminectomy.

\section{Volumetric Analysis}

The volumetric measurements preoperatively, immediate postoperatively (dogs 1 and 2) and at 12 to 36 months of follow-up are shown in - Table 2.

Immediate postoperative CT volumetric analysis of the lumbosacral lateral intervertebral neurovascular foramina in two dogs indicated a mean percentage increase in volume of $720 \%$ in extension achieved by foraminotomy (combined left and right foramen data). The increase in volume achieved by surgery ranged from 650 to $795 \%$ in the four foramina.

Follow-up CT examination performed in all six dogs at a median of 24 months postoperatively indicated a mean percentage increase in volume of $335 \%$ in extension compared with preoperative foraminal volume (combined left

Table 1 Data from six dogs diagnosed with degenerative lumbosacral stenosis that underwent lateral foraminotomy

\begin{tabular}{|l|l|l|l|l|l|}
\hline Dog & Breed & $\begin{array}{l}\text { Age } \\
(\mathbf{y})\end{array}$ & Sex & $\begin{array}{l}\text { Weight } \\
(\mathbf{k g})\end{array}$ & History \\
\hline 1 & Rottweiler & 3 & M & 39 & $\begin{array}{l}\text { 5-mo history of hindlimb stiffness. Severe difficulty jumping into } \\
\text { vehicles, scaling walls/fences/lame after jumping }\end{array}$ \\
\hline 2 & $\begin{array}{l}\text { GSD } \\
\text { (PDS) }\end{array}$ & 8 & M & 39 & $\begin{array}{l}\text { 3-y history of mild lameness after heavy work, lordosis and } \\
\text { lumbosacral epaxial muscle pain. Mild urinary incontinence for several } \\
\text { weeks. Recent poor work performance, reluctance to jump, apparent } \\
\text { pain and bunny hopping gait }\end{array}$ \\
\hline 3 & Beagle & 7 & FS & 14.5 & $\begin{array}{l}\text { 2-mo history of back pain after a traumatic incident. Intermittent } \\
\text { exercise induced left hind lameness. Hunched back and abnormal tail } \\
\text { carriage }\end{array}$ \\
\hline 4 & GSD x Labrador & 6 & M & 31 & $\begin{array}{l}\text { 2-mo history of back pain, intermittent right hind lameness and } \\
\text { difficulty jumping, reluctance to exercise. 18-mo history of } \\
\text { incontinence }\end{array}$ \\
\hline 5 & Boxer & 4 & MN & 30 & $\begin{array}{l}\text { 2-mo history of progressively worsening right hind lameness, } \\
\text { difficulty rising and reluctance to jump into the back of a truck for 5 } \\
\text { mo. Resting the leg when standing }\end{array}$ \\
\hline 6 & $\begin{array}{l}\text { GSD } \\
\text { (PDS) }\end{array}$ & 3 & FS & 33.5 & $\begin{array}{l}\text { 3-mo history of back pain, difficulty jumping, right hindlimb } \\
\text { lameness, poor work performance }\end{array}$ \\
\hline
\end{tabular}

Abbreviations: F, female; FS, female spayed; GSD, German Shepherd Dog; M, male; MN, male neutered; PDS, New Zealand Police Dog Section. 


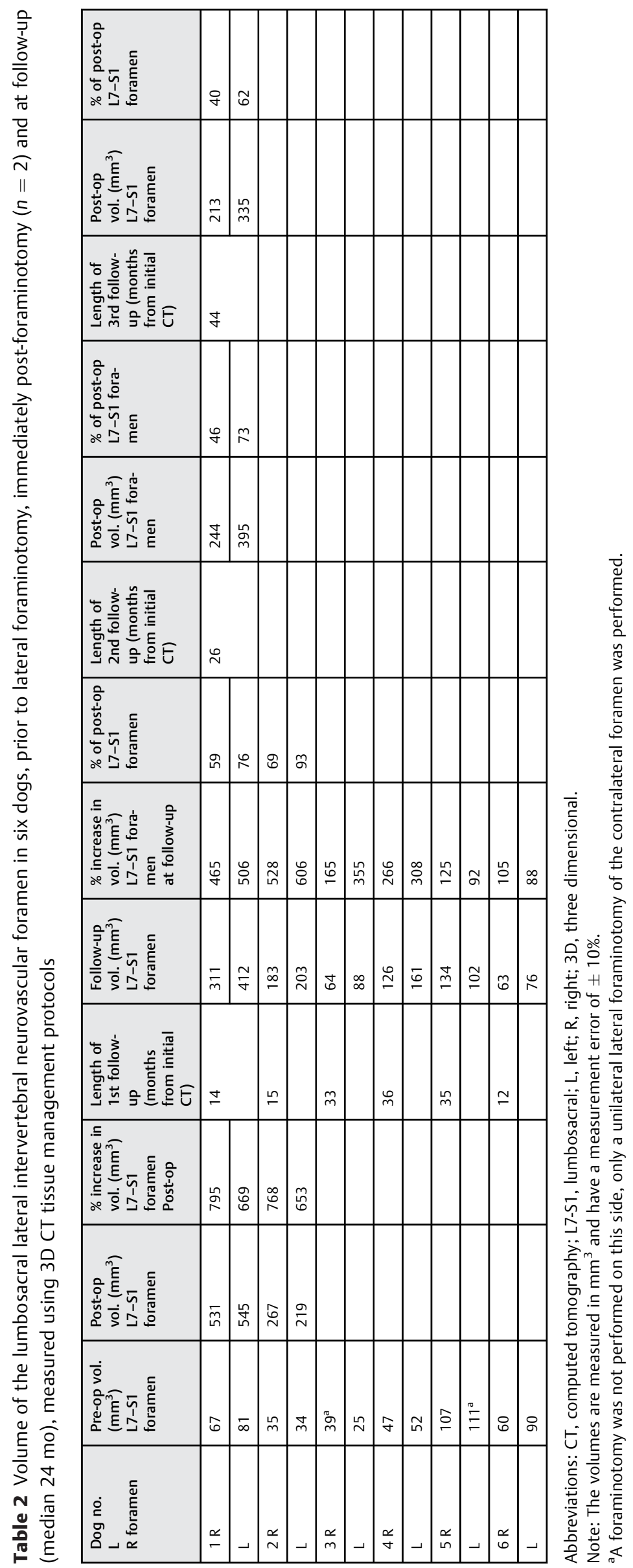


and right foraminotomy data, dog). The persistent increase in volume compared with preoperative volume ranged from 105 to $606 \%$. One foramen in one $\operatorname{dog}(\operatorname{dog} 6)$ had a smaller volume at follow-up.

In the two dogs with both immediate postoperative and follow-up CT data (14 and 15 months), the foraminal volume had reduced to a mean of $75 \%$ of that achieved by surgery (range: 59-93\%). Stated another way, modelling of the lateral intervertebral neurovascular foramina resulted in a reduction in volume by 7 to $41 \%$ (mean: $25 \%$ ) from that achieved immediately after surgery. In dog 1 , a second follow-up CT indicated a further reduction of $13 \%$ of the neuroforaminal volume on the left and a $2 \%$ reduction on the right, from 14 to 26 months. A third follow-up CT at 44 months indicated a further reduction of $6 \%$ of the neuroforaminal volume on the left and an 11\% reduction on the right, from 26 to 44 months (-Fig. 1). In five dogs, the foraminal volume of the operated lateral intervertebral neurovascular foramen was larger than its preoperative volume.

\section{Clinical and Owner Evaluation}

Five out of six dogs (83\%) returned to either full $(n=4)$ or near-full ( $n=1$ ) levels of activity according to their owners' assessment (-Appendix Table 1). One of the two Police dogs returned to work but with some limitations on performance. The other $(\operatorname{dog} 6)$ had a recurrence of clinical signs and following reevaluation with CT at 12 months post-foraminotomy underwent a dorsal stabilization procedure using a custom 3D printed titanium plate and subsequently returned to active duty. The owners of the four pet dogs with successful outcomes commented on how active their dogs were, returning to activities such as jumping and running for extended periods, free of apparent pain or lameness. Two owners commented that the recovery time after surgery was long (6 and 8 months) before their dogs fully returned to normal. Dog 5 recovered well initially, but it was then represented with the complaint of pelvic limb stiffness and an apparent return of clinical signs. Physical examination revealed bilateral cranial cruciate rupture with no signs
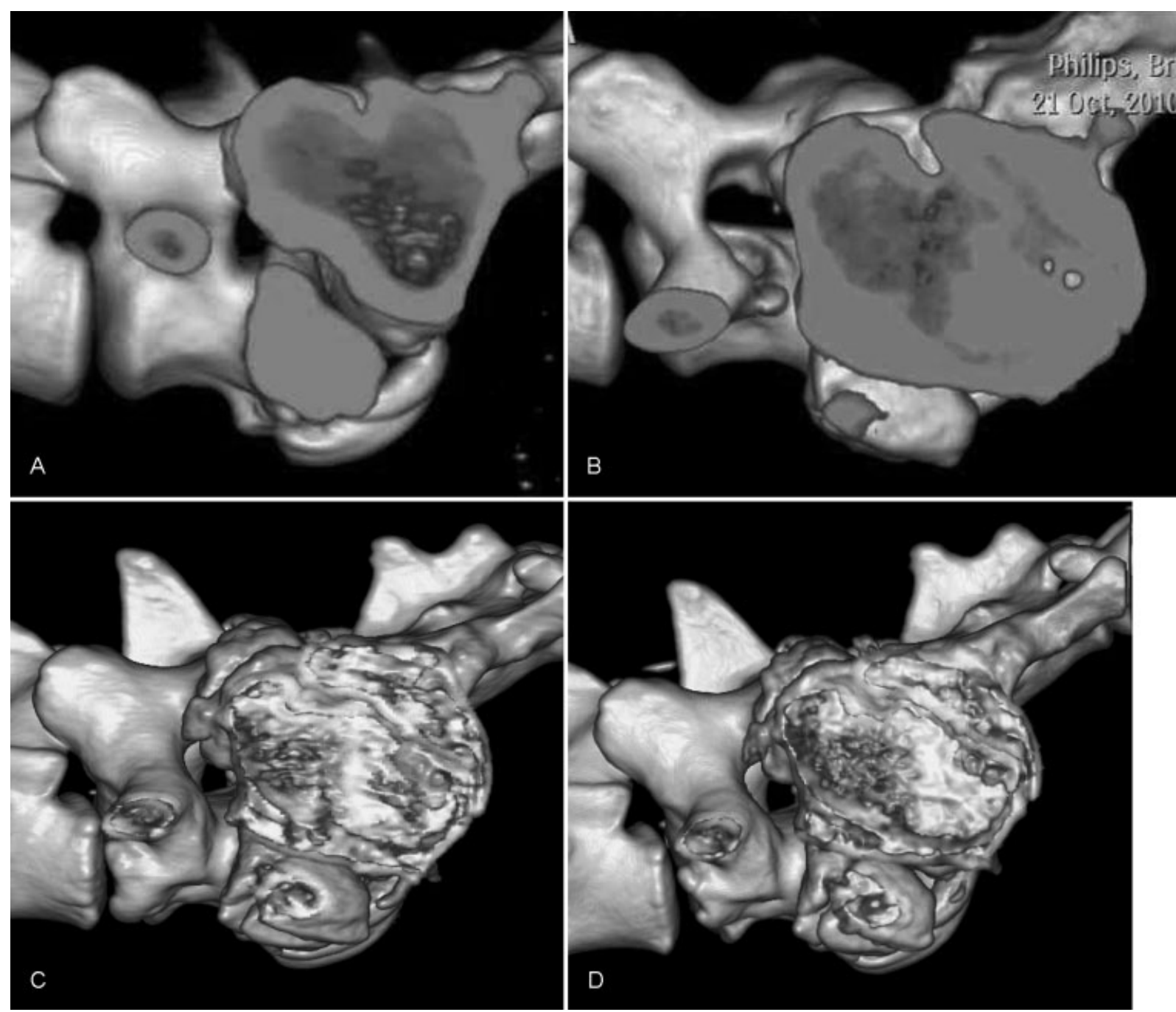

Fig. 1 Reconstructed 3D CT images of the left lumbosacral lateral intervertebral neurovascular foramen of dog no. 1 (A) preoperatively, (B) immediately postoperatively following lateral foraminotomy, (C) then 14 months and (D) 26 months postoperatively. In this particular case, the foraminotomy inadvertently extended into the dorsocaudal aspect of the L7 transverse process on the featured side. 
referable to the lumbosacral junction. The dog subsequently improved with conservative management and was free of clinical signs that the owners perceived to be referable to the lumbosacral junction. It was thus included as a successful outcome.

\section{Discussion}

Lateral foraminotomy, as performed for degenerative lumbosacral stenosis, is intended to decompress the $L 7$ nerve roots passing through the lumbosacral lateral intervertebral neurovascular foramen. Measuring foraminal volume using CT allowed the effect of lateral foraminotomy of the lumbosacral junction to be quantified. Immediate postoperative CT volumetric measurement in two dogs (four foramina) confirmed that a lateral foraminotomy procedure on average increased the volume of the lumbosacral lateral intervertebral neurovascular foramen to greater than seven times its initial volume. The degree of postoperative foraminal enlargement is, of course, entirely surgeon dependent. Regrowth of bone then occurred at the foraminotomy site in both dogs, despite which they had excellent outcomes and no recurrence of clinical signs at follow-up. It is important to note that CT volumetric analysis pre- and post-foraminotomy does not directly measure the degree of L7 compression (and any attenuation), as it defines the foraminal bone architecture only. MRI would be required to document the effect of foraminotomy at reducing L7 nerve compression more directly.

In all dogs, foraminotomy was initially successful at relieving clinical signs and had a sustained effect in five out of six dogs. The owners' assessment was based on their perception of the dogs' improved mobility and reduction in perceived pain and was therefore potentially biased due to their decision to consent to surgery. However, clinical improvement was associated with an increase in foraminal volume at follow-up that was on average three times greater than the lateral intervertebral neurovascular foraminal volume prior to surgery. Increased foraminal volume was still evident in the three dogs with greater than 30-month follow-up. However, in one of those, the increase was only $125 \%$ of preoperative volume, and given the limitations of measurement accuracy using volumetric analysis, the lateral intervertebral neurovascular foraminal volume in this dog should be considered to be approaching preoperative volume. In the one dog that had a CT at 14, 26 and 44 months postoperatively, there was a further reduction in foraminal volume between each of these scans, but the reduction represented only a quarter of the initial reduction from 0 to 12 months of follow-up, suggesting that over time modelling at the foraminotomy site abated. The recurrence of clinical signs in one working $\operatorname{dog}(\operatorname{dog} 6)$ correlated with lack of persistence of foraminal enlargement and this dog subsequently improved with stabilization of the LS junction at a neutral to slightly flexed lumbosacral angle, thus opening up the lateral intervertebral neurovascular foramina, suggesting modelling of the foraminotomy site as the probable cause for the recurrence of signs.
The technique of volumetric analysis of CT images employed has limitations. Volumetric analysis from CT using the chosen windowing pre-sets will only delineate the osseous perimeter of the foramen without the inclusion of any soft-tissue structures. Hypertrophic soft tissues can also be responsible for nerve root compression; therefore, the method described potentially overestimates the in vivo volume of the neurovascular foramen, and is not a surrogate for nerve compression per se. Measurement of the crosssectional area of the narrowest part of the foramen would be a better estimation of lateral intervertebral neurovascular foraminal narrowing, but such a method has not been found to be repeatable by bi-oblique advanced imaging ${ }^{23}$ nor when attempted by orienting and sectioning the CT volumes as described here. ${ }^{22}$ The foraminal volume is affected by the position of the lumbosacral junction. To accurately compare the lateral intervertebral neurovascular foramina volume at different time points, the dog needs to be repositioned in exactly the same positon for each scan. Although a standardized positioning protocol was used, it is not feasible for the positioning to be $100 \%$ repeatable. This variance in positioning of the patient will affect comparisons in volumetric measurements over different time points. The method of volumetric measurement used requires a rectangular region of interest to be placed over the lateral intervertebral neurovascular foramen. The width of the rectangle was set at $4 \mathrm{~mm}$ for the one smaller $\operatorname{dog}(\operatorname{dog} 3)$ and $5 \mathrm{~mm}$ for the larger dogs. It was not possible to have complete consistency in positioning the region of interest between scans, introducing a further source of error. Additionally, an increase in overall volume may not equate to effective foraminotomy if restrictive areas are not addressed at surgery. For example, a large amount of the lateral pedicle of $\mathrm{L} 7$ could be removed without breaking though the inner cortex into the lateral recess. The postoperative CT volume of the foramen would be measurably increased, but the neurovascular bundle would remain compressed due to the remaining inner cortex of the pedicle.

Regrettably, there was insufficient funding to perform a CT on all dogs immediately postoperatively. This led to only limited data on the immediate effect of lateral foraminotomy being available. Without the immediate postoperative CT data on four of the dogs, the data are relative only to the preoperative volume of the lateral intervertebral neurovascular foramen. However, this study is the first to demonstrate a persistent effect of foraminotomy. Bone modelling in the two dogs assessed immediately postoperatively suggests that the effectiveness of foraminotomy in the longer term may be lessened. A larger prospective study with more dogs undergoing both pre- and postoperative imaging is needed to confirm these preliminary findings.

An additional benefit of immediate postoperative CT examination was the feedback it provided to the surgeon on the degree of decompression achieved and an understanding of the relevant anatomy. Performing an immediate postoperative CT scan is recommended for surgeons gaining experience with lateral foraminotomy, as spatial awareness may be improved by immediate feedback. 


\section{Conclusion}

This study is the first to quantify the extent and effectiveness of the lateral foraminotomy technique at increasing the volume of the lumbosacral lateral intervertebral neurovascular foramen in the medium term. Bone modelling did occur, but the volume of the foramen 24 months postoperatively was still substantially greater than the presurgical volume in all but two of the operated dogs. It is not known whether the lateral intervertebral neurovascular foramen will continue to remodel in the longer term, reducing the effect of lateral foraminotomy. Lateral foraminotomy is a complex procedure that requires an understanding of the anatomy and appearance especially of the exit zone. Though necessarily subjective, the authors recommend that the foraminotomy be of a size that mitigates the potential effects of the modelling of bone to provide sustained decompression of the $L 7$ nerve roots and intervertebral blood vessels. Due to the immediate feedback it provides, surgeons gaining experience with lateral foraminotomy may find immediate postoperative CT imaging useful to critique their technique.

\section{Conflict of Interest}

The funding agencies played no role in the study design nor in the collection, analysis and interpretation of data, nor in the decision to submit the manuscript for publication. None of the authors has any financial or personal relationships that could inappropriately influence or bias the content of the article.

\section{Author Contributions}

Conception of study: A. Worth; Study design: A. Worth, A. Hartman, J. Bridges, B. Jones, J. Mayhew; Acquisition of data: A. Worth, A. Hartman; Data analysis and interpretation: A. Worth, A. Hartman, J. Bridges, B. Jones, J. Mayhew; Drafting or revising of manuscript: A. Worth, B. Jones, J. Mayhew.

\section{Acknowledgments}

This study was supported, in part, by a grant from the Building Research Capabilities in Strategically Relevant Areas (BRCSRA, NZ Government) Staff Development Fund and the Massey University Working Dog Centre. We wish to thank Nicola Moffat, Lee Hill, Tania Jobson and Sharyn Bray of the Massey University Veterinary Teaching Hospital Imaging Service for their technical assistance.

\section{References}

1 Chambers JN. Degenerative lumbosacral stenosis in dogs. Vet Med Rep 1989:166-180

2 De Risio L, Thomas WB, Sharp NJ. Degenerative lumbosacral stenosis. Vet Clin North Am Small Anim Pract 2000;30(01): 111-132, vi

3 Worth AJ, Thompson DJ, Hartman AC. Degenerative lumbosacral stenosis in working dogs: current concepts and review. N Z Vet J 2009;57(06):319-330
4 Meij BP, Bergknut N. Degenerative lumbosacral stenosis in dogs. Vet Clin North Am Small Anim Pract 2010;40(05):983-1009

5 Palmer RH, Chambers JN. Canine lumbosacral diseases. Part II. Definitive diagnosis, treatment and prognosis. Compend Contin Educ Pract Vet 1991;13:213-222

6 Suwankong N, Meij BP, Voorhout G, de Boer AH, Hazewinkel HA. Review and retrospective analysis of degenerative lumbosacral stenosis in 156 dogs treated by dorsal laminectomy. Vet Comp Orthop Traumatol 2008;21(03):285-293

7 Slocum B, Devine T. L7-S1 fixation-fusion for treatment of cauda equina compression in the dog. J Am Vet Med Assoc 1986; 188(01):31-35

8 Meheust P, Mallet C, Marouze C. A new surgical technique for lumbosacral stabilization: arthrodesis using the pedicle screw fixation. Anatomical aspects. Pratique Medicale et Chirurgicale de L Animal de Compagnie 2000;3:193-199

9 Bagley RS. Surgical stabilisation of the lumbosacral joint. In: Slatter DH, ed. Textbook of Small Animal Surgery, 3rd ed. Philadelphia, PA: Saunders; 2003:1238-1243

10 Denny HR, Gibbs C, Holt PE. The diagnosis and treatment of cauda equina lesions in the dog. J Small Anim Pract 1982; 23:425-443

11 Ness MG. Degenerative lumbosacral stenosis in the dog: a review of 30 cases. J Small Anim Pract 1994;35:185-190

12 Danielsson F, Sjöström L. Surgical treatment of degenerative lumbosacral stenosis in dogs. Vet Surg 1999;28(02):91-98

13 Janssens LAA, Moens Y, Coppens P, et al. Lumbosacral degenerative stenosis in the dog: the results of dorsal decompression with dorsal annulectomy and nuclectomy. Vet Comp Orthop Traumatol 2000;13:97-103

14 Hankin EJ, Jerram RM, Walker AM, King MD, Warman CG. Transarticular facet screw stabilization and dorsal laminectomy in 26 dogs with degenerative lumbosacral stenosis with instability. Vet Surg 2012;41(05):611-619

15 Golini L, Kircher PR, Lewis FI, Steffen F. Transarticular fixation with cortical screws combined with dorsal laminectomy and partial discectomy as surgical treatment of degenerative lumbosacral stenosis in 17 dogs: clinical and computed tomography follow-up. Vet Surg 2014;43(04):405-413

16 Gödde T, Steffen F. Surgical treatment of lumbosacral foraminal stenosis using a lateral approach in twenty dogs with degenerative lumbosacral stenosis. Vet Surg 2007;36(07):705-713

17 Jeffery ND, Barker A, Harcourt-Brown T. What progress has been made in the understanding and treatment of degenerative lumbosacral stenosis in dogs during the past 30 years? Vet J 2014; 201(01):9-14

18 Lee CK, Rauschning W, Glenn W. Lateral lumbar spinal canal stenosis: classification, pathologic anatomy and surgical decompression. Spine 1988;13(03):313-320

19 Wood BC, Lanz OI, Jones JC, Shires PK. Endoscopic-assisted lumbosacral foraminotomy in the dog. Vet Surg 2004;33(03):221-231

20 Kinzel S, Koch J, Stopinski T, et al. Cauda equina compression syndrome (CECS): retrospective study of surgical treatment with partial dorsal laminectomy in 86 dogs with lumbosacral stenosis. Berl Munch Tierartzl Wochenshr 2004;117(7-8):334-340

21 Chen Q Baba H, Kamitani K, Furusawa N, Imura S. Postoperative bone re-growth in lumbar spinal stenosis. A multivariate analysis of 48 patients. Spine 1994;19(19):2144-2149

22 Worth AJ, Hartman A, Bridges JP, Jones BR, Mayhew JI. Computed tomographic evaluation of dynamic alteration of the canine lumbosacral intervertebral neurovascular foramina. Vet Surg 2017;46(02):255-264

23 Higgins BM, Cripps PJ, Baker M, Moore L, Penrose FE, McConnell JF. Effects of body position, imaging plane, and observer on computed tomographic measurements of the lumbosacral intervertebral foraminal area in dogs. Am J Vet Res 2011;72(07):905-917 